\title{
Esomeprazole- or rabeprazole-based triple therapy eradicated Helicobacter pylori comparably regardless of clarithromycin susceptibility and CYP2C19 genotypes
}

\author{
Tadayoshi Okimoto,* Kazuhiro Mizukami, Ryo Ogawa, Kazuhisa Okamoto, Mitsutaka Shuto, Kensuke Fukuda, \\ Masaaki Kodama and Kazunari Murakami
}

Department of Gastroenterology, Faculty of Medicine, Oita University, 1-1 Idaigaoka, Hasama-machi, Yufu, Oita 879-5593, Japan

(Received 16 February, 2016; Accepted 8 March, 2016; Published online 16 July, 2016)

The aim of this study was to assess the efficacy of esomeprazolebased triple therapy compared with rabeprazole-based triple therapy according to CYP2C19 genotype and clarithromycin susceptibility status for first-line eradication therapy of Helicobacter pylori $(H$. pylori) in Japan. We enrolled $219 H$. pylori-infected patients, and randomly allocated patients to the EAC group (esomeprazole $20 \mathrm{mg}$, clarithromycin $200 \mathrm{mg}$, amoxicillin $750 \mathrm{mg}$ for one week, with all drugs given twice daily) or RAC group (rabeprazole $10 \mathrm{mg}$, clarithromycin $200 \mathrm{mg}$, amoxicillin $750 \mathrm{mg}$ for one week, with all drugs given twice daily). The $H$. pylori eradication rate according to the PP analyses was $75.0 \%(95 \% \mathrm{Cl}$ : $65.2-82.8 \%)$ in the EAC group and $71.4 \%(95 \% \mathrm{Cl}: 61.4-79.1 \%)$ in the RAC group. There were no statistically significant differences. The eradication rates of the clarithromycin-resistant/-sensitive strains were, respectively, $45.0 \%$ (95\% Cl: $30.7-60.2 \%) / 98.0 \%$ (95\% Cl: $88.7-100 \%)$ in the EAC group and $39.5 \%$ (95\% Cl: $25.6-$ $55.3 \%) / 93.5 \%(95 \% \mathrm{Cl}: \mathbf{8 1 . 9 - 9 8 . 4 \% )}$ in the RAC group. The eradication rate of the clarithromycin-sensitive strains was significantly higher than that of the resistant strains in both groups. In conclusion, EAC and RAC therapies show a comparable efficacy regardless of the CYP2C19 genotype and clarithromycin susceptibility status in Japan.

Key Words: Helicobacter pylori, eradication, esomeprazole, rabeprazole, CYP2C19

Helicobacter pylori (H. pylori) infection is known to cause - various upper gastrointestinal (GI) diseases, including atrophic gastritis, gastroduodenal ulcer, mucosa-associated lymphoid tissue (MALT) lymphoma and gastric cancer. ${ }^{(1-3)}$ Eradication of $H$. pylori infection has been reported to be an effective therapy to prevent or cure H. pylori-associated diseases. One standard eradication therapy is the combination of a proton pump inhibitor (PPI) with clarithromycin (CAM) and amoxicillin (AMPC). This first-line triple therapy has been covered under Japan's national health insurance (NHI) scheme for patients with gastric or duodenal ulcers since 2000, and its indication was expanded for $H$. pylori-infected atrophic gastritis in 2013. In recent years, decreasing primary eradication rates have been reported. ${ }^{(4)}$ The primary cause of this decrease is an increase in CAM-resistant strains of $H$. pylori, although it is also possible that the effect of the PPIs may be insufficient. PPIs are widely used for the prevention and treatment of acid-related diseases. Acid inhibition increases eradication efficacy because antibiotics are more stable in a less-acidic gastric condition, which induces a higher antibiotic sensitivity to the bacteria, and PPIs are more efficient in inhibiting gastric acid secretion than histamine $(\mathrm{H} 2)$ receptor antagonists. ${ }^{(5)}$ However, a disadvantage of the acid inhibitory effect of PPIs is the effect of CYP2C19, a hepatic drugmetabolizing enzyme that degrades PPIs. It is well known that CYP2C19 has polymorphisms in exon $4(* 3)$ and exon $5(* 2)$. Furthermore, the homozygous extensive metabolizer (HomoEM) has two wild-type alleles $(* 1 / * 1)$, heterozygous EM (HeteroEM) has one loss-of function (LOF) variant allele $(1 * / 2 *$ or $1 * / 3 *)$, and poor metabolizer $(\mathrm{PM})$ has two LOF variant alleles $(* 2 / * 2, * 2 / * 3$, $* 3 / * 3){ }^{(6)}$

In patients with high CYP2C19 enzyme (EM) activity, the effect of PPIs is not sufficiently strong due to enzymatic degradation. Second-generation PPIs have been developed to address these disadvantages. Rabeprazole is a second-generation PPI that is not strongly influenced by CYP2C19 enzyme activity because its main metabolizing enzyme is not CYP2C19. ${ }^{(6,7)}$ The secondgeneration PPI esomeprazole is the S-isomer of omeprazole and is also not strongly influenced by CYP2C19 enzyme activity. ${ }^{(8,9)}$ Furuta et al. ${ }^{(10)}$ reported the intra gastric $\mathrm{pH}$ after oral administrations of esomeprazole and rabeprazole. It was shown that esomeprazole and rabeprazole were similarly effective regardless of CYP2C19 genotypes. Meta-analysis of $H$. pylori eradication rates comparing esomeprazole or rabeprazole to first-generation PPIs including omeprazole and lansoprazole demonstrated that esomeprazole and rabeprazole show better overall $H$. pylori eradication rates than first-generation PPIs. ${ }^{(11)}$ It was shown that rabeprazole-based triple therapy achieves similar $H$. pylori eradication rates to esomeprazole in first-line triple therapy including CAM and AMPC in Taiwan. ${ }^{(12)}$

However, a randomized trial comparing the efficacy of esomeprazole and rabeprazole-based regimens according to CYP2C19 genotype in Japan has not been conducted to date. The aim of this study was to compare the efficacy of these two PPI-based regimens according to CYP2C19 genotype status in the first-line eradication therapy of $H$. pylori infection in Japan.

\section{Materials and Methods}

Patients and study design. This was a Japanese multicenter, prospective, randomized, controlled study. Patients were enrolled at 6 hospitals: Oita University Hospital, Arita GI Hospital,

*To whom correspondence should be addressed.

E-mail: okimoto@oita-u.ac.jp 
Tsurumi Hospital, Almeida Memorial Hospital, JCHO Nankai Medical Center, and Usatakada Medical Association Hospital. A total of 219 patients (110 men, 109 women; age: $57.458 .3 \pm$ 13.513.7 years, mean \pm SD) referred to us between April 2012 and May 2013 were enrolled. At entry, a patient was diagnosed as H. pylori-positive if a rapid urease test was positive. Exclusion criteria included (1) patients with previous therapy for $H$. pylori, (2) ingestion of antibiotics, bismuth or PPI within the prior 2 weeks, (3) alcohol or drug addiction, (4) allergic history to the medications used in this study, (5) the coexistence of serious concomitant illness, (6) pregnant women, and (7) previous partial or total gastrectomy. Patients were randomly assigned by computer to one of the following two eradication groups which are recommended in Japanese guidelines: (1) EAC group: esomeprazole $20 \mathrm{mg}$, CAM $200 \mathrm{mg}$, AMPC $750 \mathrm{mg}$ for one week, with all drugs given twice daily; (2) RAC group: rabeprazole $10 \mathrm{mg}$, CAM $200 \mathrm{mg}$, AMPC $750 \mathrm{mg}$ for one week, with all drugs given twice daily. The determination of eradication was performed using a urea breath test (UBT) from 4 to 8 weeks after completing the eradication therapy. Measured values were expressed as $\Delta^{13} \mathrm{C} \%$ at $20 \mathrm{~min}$ after administration with a cut-off of $\Delta 2.5 \%$. Subjects with $\geq \Delta 2.5 \%$ o were considered positive for $H$. pylori, and those with $<\Delta 2.5 \%$ were considered negative. For drug susceptibility test, endoscopic biopsy specimens were obtained from the greater curvature of the body and the antrum of the stomach.

The study was performed according to the Declaration of Helsinki and was approved by each institution's ethics committee. This trial is registered with UMIN Clinical trials: http://www. umin.ac.jp/ctr/, number UMIN000007550. All of the participants gave written informed consent.

Drug susceptibility test. Antimicrobial susceptibility was determined using the epsilometer test (E-test). The breakpoints were set at $1,1,1$, and $16 \mu \mathrm{g} / \mathrm{ml}$ for CAM, AMPC, levofloxacin (LVFX) and metronidazole (MNZ), respectively, and a strain was deemed to be resistant when their MIC values were greater than or equal to the breakpoint.

CYP2C19 genotyping. Blood samples for the determination of CYP2C19 genotype were collected. DNA was isolated from whole blood using a WIAamp DNA Blood Mini Kit (Qiagen Inc., Valencia, CA). Real-time polymerase chain reaction was used for the detection of the wild-type CYP2C19*1 gene and the two mutated genes, CYP2C19*2 and CYP2C19*3, using a specific TaqMan SNP Genotyping assay (Applied Biosystems, Foster, CA) and a LightCyclerR 480 Real-Time PCR System (Roche Diagnostics, Basel, Switzerland). ${ }^{(13,14)}$ Homozygous EM was defined as wt/wt, heterozygous EM was defined as $\mathrm{wt} / \mathrm{m} 1$ and $\mathrm{wt} / \mathrm{m} 2$, and PM was defined as $\mathrm{m} 1 / \mathrm{m} 1, \mathrm{~m} 2 / \mathrm{m} 2$ and $\mathrm{m} 1 / \mathrm{m} 2$.

Statistical analysis. The eradication rate was evaluated by the intention-to-treat (ITT) and per protocol (PP) analysis. The ITT analysis accounted for all patients enrolled in the study. Patients who did not have their final UBT results were regarded as failure cases. Patients that were not administered the UBT due to loss to follow-up were excluded from the PP analysis. We calculated the sample size based on a non-inferiority margin of $10 \%$, a successful eradication rate of at least $77 \%$, a two-sided test at the $5 \%$ level, and a power of $90 \%$. Based on this, a sample size of 105 patients per therapy group was calculated to be sufficient. We decided to increase the number to 110 patients per therapy group to compensate for a $5 \%$ loss at follow-up. Statistical analysis of intergroup differences in eradication rates was performed using the chi-squared test. Between-group comparisons of patient characteristics (sex, age, resistance rate, and CYP2C19 genotype) were conducted using the chi-squared test, Fisher's exact test and Student's $t$ test as appropriate. Statistical significance was set at $p$ values less than 0.05 . Statistical analyses were performed using SPSS software (PASW Statistics 18, SPSS Japan).

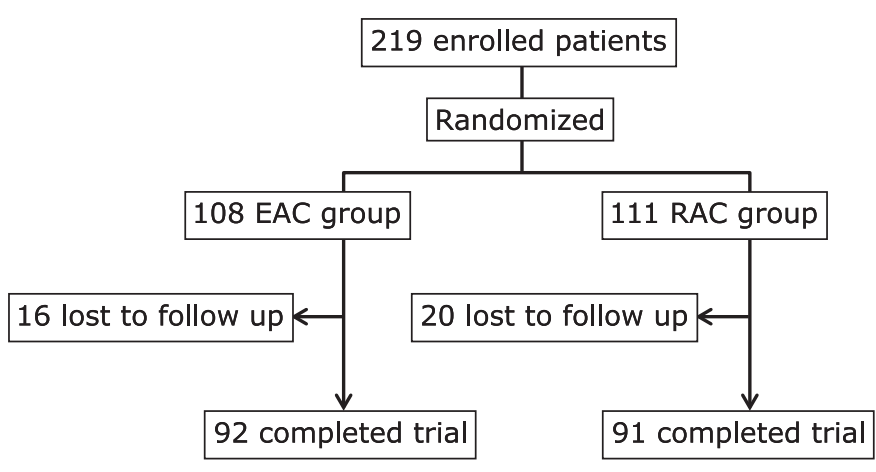

Fig. 1. Study flow chart. Patients were randomly assigned the patients to the EAC group $(n=108)$ or RAC group $(n=111)$. Sixteen patients in the EAC group and 20 patients in the RAC group were lost to follow-up. EAC, esomeprazole in combination with amoxicillin and clarithromycin; RAC, rabeprazole in combination with amoxicillin and clarithromycin.

\section{Results}

Patients. A total of 219 patients evaluated at 6 hospitals were enrolled from April 2012 to May 2013. We randomly assigned the patients to the EAC group $(n=108)$ or RAC group $(n=111)$. After randomization, 16 patients in the EAC group and 20 patients in the RAC group were lost to follow-up (Fig. 1). The mean age of the study population was $57.4 \pm 13.5$ years; there were 110 men $(50.2 \%)$. There were no significant differences between the two treatment groups with respect to patient demographics, clinical characteristics, drug resistance or CYP2C19 genotype pattern (Table 1). Both treatment groups achieved $>90 \%$ drug compliance.

Eradication of $\boldsymbol{H}$. pylori infection. The eradication rates of the two treatment groups are shown in Table 2. ITT analysis showed eradication rates of $63.9 \%(69 / 108,95 \%$ CI: $54.5-72.3 \%)$ for the EAC group and 58.6\% (65/111, 95\% CI: 49.3-67.3\%) for the RAC group. PP analysis showed eradication rates of $75.0 \%$ (69/92, 95\% CI: $65.2-82.8 \%$ ) for the EAC group and $71.4 \%$ (65/91, 95\% CI: $61.4-79.7 \%)$ for the RAC group. The eradication rates between the two treatment groups were not significantly different as determined by ITT and PP analysis (ITT, $p=0.42$; PP, $p=0.59$ ).

Eradication rates based on the genotypic differences of CYP2C19. Fig. 2 shows the eradication rates based on the CYP2C19 genotypic differences using PP analysis. The eradication rates determined by PP analysis were $77.3 \%$ (17/22; homozygous EM), 75.5\% (37/49; heterozygous EM), and 71.4\% (15/21; PM) for the EAC group and $69.7 \%$ (23/33; homozygous EM), $74.4 \%$ (29/39; heterozygous EM), and $68.4 \%$ (13/19; PM) for the RAC group. No statistically significant differences were observed.

Eradication rates based on the CAM susceptibility test.

Among the 219 enrolled patients, we performed cultures and drug susceptibility tests of $210 \mathrm{H}$. pylori strains. The resistance rates to CAM, MNZ and LVFX were $40.5 \%(85 / 210), 3.8 \%$ $(8 / 210)$ and $54.8 \%(115 / 210)$, respectively. There was no significant difference between the EAC and RAC groups (Table 1). No resistance to AMPC was observed. The eradication rates for the CAM-resistant EAC and RAC groups according to PP analysis were $45.0 \%$ (18/40, 95\% CI: $30.7-60.2 \%)$ and $39.5 \%$ (15/38, 95\% CI: $25.6-55.3 \%)$, respectively. Conversely, the eradication rates for the CAM-sensitive EAC and RAC groups according to PP analysis were $98.0 \%(50 / 51,95 \%$ CI: $88.7-100 \%)$ and $93.5 \%$ (43/46, 95\% CI: 81.9-98.4\%), respectively (Fig. 3). No statistically significant differences were observed between these eradication regimens. The eradication rate for the CAM-sensitive strains was significantly higher than that of the CAM-resistant strains in 
Table 1. Summary of patients' demographic characteristics

\begin{tabular}{|c|c|c|c|c|}
\hline & $\begin{array}{l}\text { Overall } \\
(n=219)\end{array}$ & $\begin{array}{l}\text { EAC group } \\
(n=108)\end{array}$ & $\begin{array}{c}\text { RAC group } \\
(n=111)\end{array}$ & $p$ value \\
\hline $\begin{array}{l}\text { Age (years; mean } \pm S D \text { ) } \\
\text { range }\end{array}$ & $\begin{array}{l}57.4 \pm 13.5 \\
22-85\end{array}$ & $\begin{array}{l}57.6 \pm 13.4 \\
24-85\end{array}$ & $\begin{array}{l}57.1 \pm 13.6 \\
22-84\end{array}$ & $0.78^{a}$ \\
\hline $\begin{array}{l}\text { Gendar } \\
\text { Male } \\
\text { Female }\end{array}$ & $\begin{array}{l}110 \\
109\end{array}$ & $\begin{array}{l}56 \\
52\end{array}$ & $\begin{array}{l}54 \\
57\end{array}$ & $0.64^{b}$ \\
\hline $\begin{array}{l}\text { Underlying disease } \\
\text { Gastric ulcer } \\
\text { Duodenal ulcer } \\
\text { Gastric/Duodenal ulcer } \\
\text { Atrophic gastritis } \\
\text { Gastric cancer }\end{array}$ & $\begin{array}{c}37 \\
52 \\
28 \\
96 \\
6\end{array}$ & $\begin{array}{c}17 \\
29 \\
13 \\
46 \\
3\end{array}$ & $\begin{array}{c}20 \\
23 \\
15 \\
50 \\
3\end{array}$ & $0.80^{\mathrm{b}}$ \\
\hline $\begin{array}{l}\text { H. pylori resistant pattern } \\
\text { Clarithromycin } \\
\text { Metronidazole } \\
\text { Ampicillin } \\
\text { Levofloxacin }\end{array}$ & $\begin{array}{c}85 / 210(40.5 \%) \\
8 / 210(3.8 \%) \\
0 / 210 \\
115 / 210(54.8 \%)\end{array}$ & $\begin{array}{c}44 / 106(41.5 \%) \\
3 / 106(2.8 \%) \\
0 / 106 \\
59 / 106(55.6 \%)\end{array}$ & $\begin{array}{c}41 / 104(39.4 \%) \\
5 / 104(4.8 \%) \\
0 / 104 \\
56 / 104(53.8 \%)\end{array}$ & $\begin{array}{c}0.76^{\mathrm{b}} \\
0.45^{\mathrm{b}} \\
\text { ND } \\
0.79^{\mathrm{b}}\end{array}$ \\
\hline $\begin{array}{c}\text { CYP2C19 genotypes } \\
\text { Homozygous EM } \\
\text { Heterozygous EM } \\
\text { Poor metabolizers }\end{array}$ & $\begin{array}{c}71 / 219(32.4 \%) \\
103 / 219(47.0 \%) \\
45 / 219(20.5 \%)\end{array}$ & $\begin{array}{l}32 / 108(29.6 \%) \\
52 / 108(48.1 \%) \\
24 / 108(22.2 \%)\end{array}$ & $\begin{array}{l}39 / 111(35.1 \%) \\
51 / 111(45.9 \%) \\
21 / 111(18.9 \%)\end{array}$ & $\begin{array}{l}0.38^{b} \\
0.74^{b} \\
0.55^{b}\end{array}$ \\
\hline
\end{tabular}

${ }^{a} t$ test. ${ }^{b}$ chi-square test. ND, not determined; H. pylori, Helicobacter pylori; EM, extensive metabolizer.

Table 2. Eradication rates of esomeprazole- and rabeprazole-based regimen

\begin{tabular}{ccc}
\hline & \multicolumn{2}{c}{ Regimen } \\
\cline { 2 - 3 } & EAC & RAC \\
\hline ITT & $63.9 \%$ & $58.6 \%$ \\
& {$[69 / 108]$} & {$[65 / 111]$} \\
PP & $(54.5-72.3)$ & $(49.3-67.3)$ \\
& $75.0 \%$ & $71.4 \%$ \\
& {$[69 / 92]$} & {$[65 / 91]$} \\
& $(65.2-82.8)$ & $(61.4-79.7)$ \\
\hline
\end{tabular}

Eradication rates (\%) are reported with $95 \%$ confidence interval in parentheses. Numbers in square brackets are number of patients cured/ total number of patients recruited. ITT, intention-to-treat analysis; $\mathrm{PP}$, per protocol analysis; EAC, esomeprazole in combination with amoxicillin and clarithromycin; RAC, rabeprazole in combination with amoxicillin and clarithromycin.

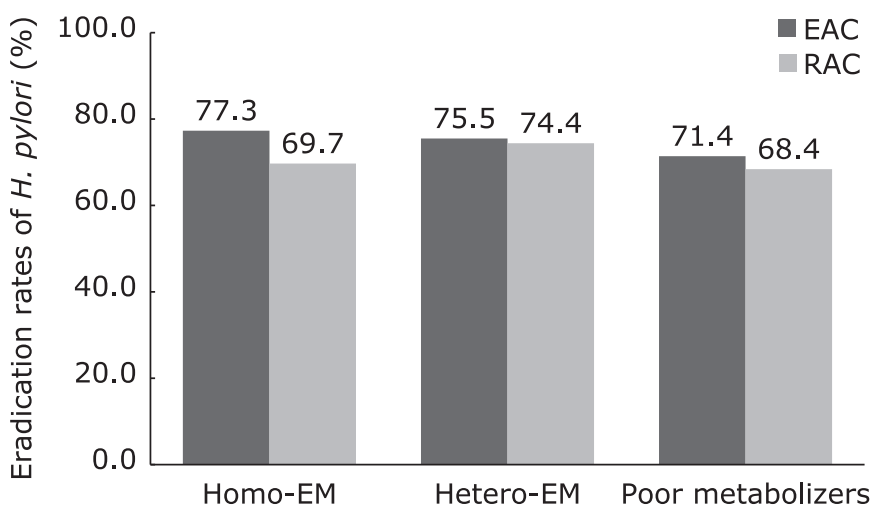

Fig. 2. Eradication rates of according to CYP2C19 genotype in the per protocol analysis. The eradication rates were compared in three CYP2C19 genotypes, Homo-EM, Hetero-EM, Poor metabolizer. Homo-EM, homozygous extensive metabolizer; Hetero-EM, heterozygous extensive metabolizer; EAC, esomeprazole in combination with amoxicillin and clarithromycin; RAC, rabeprazole in combination with amoxicillin and clarithromycin.

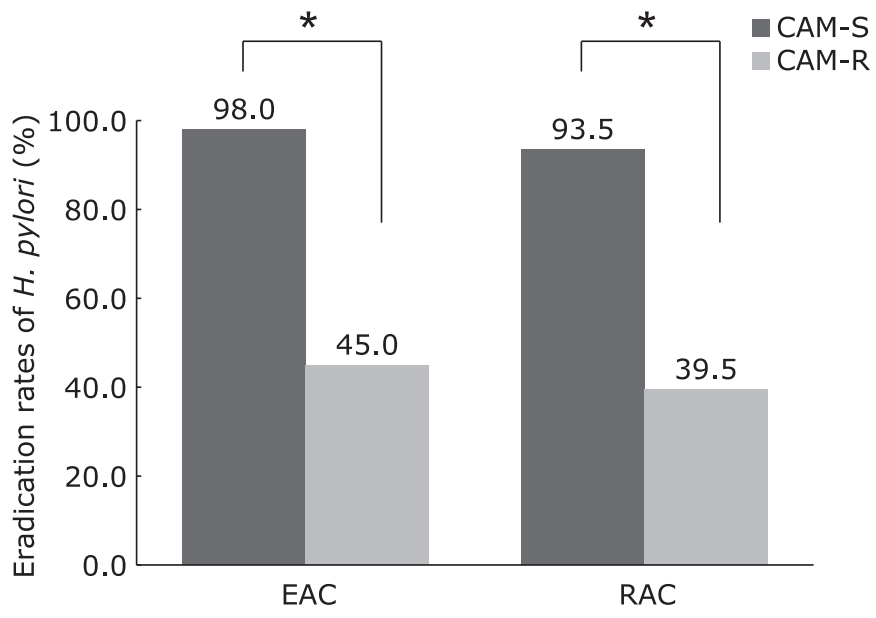

Fig. 3. Clarithromycin resistance and eradication rates in the per protocol analysis. The eradication rates were compared according to the clarithromycin susceptibility status. EAC, esomeprazole in combination with amoxicillin and clarithromycin; RAC, rabeprazole in combination with amoxicillin and clarithromycin; CAM-S, clarithromycin sensitive; CAM-R, clarithromycin resistant; ${ }^{*} p<0.001$.

both the EAC $(p<0.001)$ and RAC $(p<0.001)$ groups based on PP analysis.

\section{Discussion}

The combination of PPI, CAM and AMPC is one of the most popular $H$. pylori eradication regimens and is the only regimen approved for first-line therapy in Japan. PPIs can enhance the efficacy of antibiotics through decreased antibiotic decay within the gastric juice and increased sensitivity of $H$. pylori to antibiotics. ${ }^{(15,16)}$ Although PPIs are affected by the CYP2C19 polymorphism, to date, no information has been reported concerning esomeprazole-based combination therapy and the impact of CYP2C19 polymorphisms on H. pylori eradication rates in Japan. 
The present study compared the efficacy of esomeprazole and rabeprazole-based regimens according to the CYP2C19 genotype. Our findings confirmed that the clinical efficacy of esomeprazole and rabeprazole-based triple therapies on the eradication rates was not influenced by the CYP2C19 genotype status.

The meta-analysis by McNicholl et al. ${ }^{(11)}$ showed that secondgeneration PPIs (which are not strongly influenced by CYP2C19 enzyme activity, such as esomeprazole or rabeprazole) could achieve a higher eradication rate of $H$. pylori than first-generation PPIs (which are influenced by CYP2C19 enzyme activity, such as omeprazole or lansoprazole). In their sub-analysis, the eradication rates in patients with EM were significantly higher with the second-generation PPIs. No differences in the eradication rates in PM patients were found between first- and second-generation PPIs. Two Japanese studies reported the influence of CYP2C19 genotypes on the $H$. pylori eradication rates in patients taking rabeprazole-based triple therapies. Miki et al. ${ }^{(17)}$ observed an $H$. pylori eradication rate of $91.1 \%$ in homozygous EM, $91.3 \%$ in heterozygous EM and $76.9 \%$ in PM with no statistically significant difference. Their data also showed that CAM-R is clinically relevant in the $H$. pylori eradication rate $(\mathrm{OR}=498.3,95 \%$ $\mathrm{CI}=37.9-6,544.7)$. Hokari et al ${ }^{(18)}$ reported H. pylori eradication of $85.8 \%$ in homozygous and heterozygous EM and $76.9 \%$ in PM; this difference was not statistically significant. Lee et al. ${ }^{(19)}$ reported the eradication rate compared the efficacy of esomeprazole and rabeprazole-based regimens according to the CYP2C19 genotype. Their eradication rates were $87.0 \%$ (homozygous EM), $80.4 \%$ (heterozygous EM), and $91.7 \%$ (PM) for the EAC group and $73.9 \%$ (homozygous EM), $78.9 \%$ (heterozygous EM), and 91.3\% (PM) for the RAC group; no statistically significant difference was observed among the groups.

A major reason for failure of $H$. pylori eradication is the presence of resistance against antibiotics. Nishizawa et al. ${ }^{(20)}$ reported the efficacy of CAM or MNZ containing regimens in first-line eradication therapy. The eradication rates of CAM containing regimen was $74.5 \%$ (ITT), whereas MNZ containing regimen was $96.4 \%$. The regimen composed of PPI, MNZ and AMPC was significantly more effective than that composed of PPI, CAM and AMPC. Sasaki et al. ${ }^{(21)}$ documented an increasing trend in primary CAM resistance of $H$. pylori, rising from $8.7 \%$ (1997-2000) to $34.4 \%$ (2007-2008) in Japan. Concomitantly, H. pylori eradication rates declined from $90.6 \%$ to $74.8 \%$. Kawai et al. ${ }^{(4)}$ also reported a decreasing trend in $H$. pylori eradication rates, with $H$. pylori eradication rates significantly decreasing from $78.5 \%$ in 2001 to $66.5 \%$ in 2010 .

In this study, we showed that the prevalence of CAM resistance

\section{References}

1 Uemura N, Okamoto S, Yamamoto S, et al. Helicobacter pylori infection and the development of gastric cancer. $N$ Engl J Med 2001; 345: 784-789.

2 Wotherspoon AC, Doglioni C, Diss TC, et al. Regression of primary lowgrade B-cell gastric lymphoma of mucosa-associated lymphoid tissue type after eradication of Helicobacter pylori. Lancet 1993; 342: 575-577.

3 Nguyen TT, Kim SJ, Park JM, Hahm KB, Lee HJ. Repressed TGF- $\beta$ signaling through CagA-Smad3 interaction as pathogenic mechanisms of Helicobacter pylori-associated gastritis. J Clin Biochem Nutr 2015; 57: 113 120.

4 Kawai T, Takahashi S, Suzuki H, et al. Changes in the first line Helicobacter pylori eradication rates using the triple therapy-a multicenter study in the Tokyo metropolitan area (Tokyo Helicobacter pylori study group). J Gastroenterol Hepatol 2014; 29 Suppl 4: 29-32.

5 Calvet X, Gomollón F. What is potent acid inhibition, and how can it be achieved? Drugs 2005; 65 Suppl 1: 13-23.

6 Adachi K, Katsube T, Kawamura A, et al. CYP2C19 genotype status and intragastric $\mathrm{pH}$ during dosing with lansoprazole or rabeprazole. Aliment Pharmacol Ther 2000; 14: 1259-1266.

7 Ishizaki T, Horai Y. Review article: cytochrome P450 and the metabolism of and LVFX were higher at $40.5 \%$ and $54.8 \%$, respectively, and only CAM resistance impaired the effectiveness of the triple therapy. The choice of either esomeprazole or rabeprazole did not affect the eradication rate in Japan.

There were some limitations in this study. The eradication rates based on ITT analysis were low compared with the PP analysisderived eradication rates. The low eradication rates based on ITT analysis could be attributed to the large number of patients who received eradication therapy but failed to visit the hospital for assessment of successful eradication. Most of the dropouts occurred at one institution that was a private hospital located in the neighborhood of a commercial zone. The mean age of the dropouts was significantly lower than that of all of the enrolled patients (52.5 and 57.4 years, respectively). Therefore, it is possible that the dropouts could not visit the hospital because they were too busy with their work.

Two recent Japanese studies reported the efficacy of esomeprazole or rabeprazole-based regimens in first-line eradication therapy. Nishida et al. ${ }^{(22)}$ reported that the $H$. pylori eradication rates for EAC were $76.9 \%$ based on PP analysis and $69.4 \%$ based on ITT analysis. Regarding the RAC eradication rates, Nishizawa et al. ${ }^{(23)}$ reported that the H. pylori eradication rates for RAC were $77.2 \%$ using PP analysis and $73.3 \%$ using ITT analysis; the eradication rates based on PP analysis were 50\% (3/6) for CAM-resistant strains and $92.8 \%$ (13/14) for CAM-sensitive strains. Because of the small number of samples, unlike in our case, the difference in their study was not significant $(p=0.061)$. These eradication rates were similar to our findings, particularly in the case of PP analysis.

In conclusion, the clinical efficacy of esomeprazole and rabeprazole-based first-line eradication therapies is independent of the CYP2C19 genotype. Additionally, CAM resistance is strongly related to the failure of first-line eradication therapy.

\section{Acknowledgments}

We would like to thank Hisanori Abe (Arita GI Hospital), Takayuki Nagai (Tsurumi Hospital), Hideki Ono (Almeida Memorial Hospital), Kunimitsu Inoue (Usatakada Medical Association Hospital) and Mistuteru Motomura (JCHO Nankai Medical Cente) for collecting clinical data.

\section{Conflict of Interest}

No potential conflicts of interest were disclosed.

proton pump inhibitors--emphasis on rabeprazole. Aliment Pharmacol Ther 1999; 13 Suppl 3: 27-36.

8 Andersson T, Röhss K, Bredberg E, Hassan-Alin M. Pharmacokinetics and pharmacodynamics of esomeprazole, the S-isomer of omeprazole. Aliment Pharmacol Ther 2001; 15: 1563-1569.

9 Hassan-Alin M, Andersson T, Niazi M, Röhss K. A pharmacokinetic study comparing single and repeated oral doses of $20 \mathrm{mg}$ and $40 \mathrm{mg}$ omeprazole and its two optical isomers, S-omeprazole (esomeprazole) and R-omeprazole, in healthy subjects. Eur J Clin Pharmacol 2005; 60: 779-784.

10 Furuta K, Kohata Y, Fujiwara Y, et al. Intra-gastric $\mathrm{pH}$ following single oral administrations of rabeprazole and esomeprazole: double-blind cross-over comparison. J Clin Biochem Nutr 2014; 55: 178-183.

11 McNicholl AG, Linares PM, Nyssen OP, Calvet X, Gisbert JP. Metaanalysis: esomeprazole or rabeprazole vs. first-generation pump inhibitors in the treatment of Helicobacter pylori infection. Aliment Pharmacol Ther 2012; 36: 414-425.

12 Wu IC, Wu DC, Hsu PI, et al. Rabeprazole- versus esomeprazole-based eradication regimens for $H$. pylori infection. Helicobacter 2007; 12: 633-637.

13 Perini JA, Vargens DD, Santana IS, et al. Pharmacogenetic polymorphisms 
in Brazilian-born, first-generation Japanese descendants. Braz J Med Biol Res 2009; 42: 1179-1184.

14 Semiz S, Dujic T, Ostanek B, et al. Analysis of CYP2C9*2, CYP2C19*2, and CYP2D6*4 polymorphisms in patients with type 2 diabetes mellitus. Bosn J Basic Med Sci 2010; 10: 287-291.

15 Goddard AF, Jessa MJ, Barrett DA, et al. Effect of omeprazole on the distribution of metronidazole, amoxicillin, and clarithromycin in human gastric juice. Gastroenterology 1996; 111: 358-367.

16 Erah PO, Goddard AF, Barrett DA, Shaw PN, Spiller RC. The stability of amoxycillin, clarithromycin and metronidazole in gastric juice: relevance to the treatment of Helicobacter pylori infection. J Antimicrob Chemother 1997; 39: $5-12$.

17 Miki I, Aoyama N, Sakai T, et al. Impact of clarithromycin resistance and CYP2C19 genetic polymorphism on treatment efficacy of Helicobacter pylori infection with lansoprazole- or rabeprazole-based triple therapy in Japan. Eur J Gastroenterol Hepatol 2003; 15: 27-33.

18 Hokari K, Sugiyama T, Kato M, et al. Efficacy of triple therapy with rabeprazole for Helicobacter pylori infection and CYP2C19 genetic polymorphism. Aliment Pharmacol Ther 2001; 15: 1479-1484.
19 Lee VW, Chau TS, Chan AK, et al. Pharmacogenetics of esomeprazole or rabeprazole-based triple therapy in Helicobacter pylori eradication in Hong Kong non-ulcer dyspepsia Chinese subjects. J Clin Pharm Ther 2010; 35: $343-350$.

20 Nishizawa T, Suzuki H, Suzuki M, Takahashi M, Hibi T. Proton pump inhibitor-amoxicillin-clarithromycin versus proton pump inhibitor-amoxicillinmetronidazole as first-line Helicobacter pylori eradication therapy. J Clin Biochem Nutr 2012; 51: 114-116.

21 Sasaki M, Ogasawara N, Utsumi K, et al. Changes in 12-year first-line eradication rate of Helicobacter pylori based on triple therapy with proton pump inhibitor, amoxicillin and clarithromycin. J Clin Biochem Nutr 2010; 47: 5358.

22 Nishida T, Tsujii M, Tanimura H, et al. Comparative study of esomeprazole and lansoprazole in triple therapy for eradication of Helicobacter pylori in Japan. World J Gastroenterol 2014; 20: 4362-4369.

23 Nishizawa T, Maekawa T, Watanabe N, et al. Clarithromycin versus metronidazole as first-line Helicobacter pylori eradication: a multicenter, prospective, randomized controlled study in Japan. J Clin Gastroenterol 2015; 49: $468-471$. 